\title{
MMBERT: Multimodal BERT Pretraining for Improved Medical VQA
}

\author{
Yash Khare $^{\star \dagger} \quad$ Viraj Bagal ${ }^{\star \ddagger} \quad$ Minesh Mathew ${ }^{\dagger}$ \\ Adithi Devi ${ }^{\dagger \dagger} \quad$ U Deva Priyakumar ${ }^{\dagger} \quad$ CV Jawahar ${ }^{\dagger}$ \\ $\dagger$ IIIT Hyderabad, India $\quad{ }^{\ddagger}$ IISER Pune, India $\quad{ }^{\dagger \dagger}$ Osmania Medical College, India
}

\begin{abstract}
Images in the medical domain are fundamentally different from the general domain images. Consequently, it is infeasible to directly employ general domain Visual Question Answering (VQA) models for the medical domain. Additionally, medical images annotation is a costly and timeconsuming process. To overcome these limitations, we propose a solution inspired by self-supervised pretraining of Transformer-style architectures for NLP, vision and Language tasks. Our method involves learning richer medical image and text semantic representations using Masked Language Modeling (MLM) with image features as the pretext task on a large medical image+caption dataset. The proposed solution achieves new state-of-the-art performance on two VQA datasets for radiology images - VQA-Med 2019 and VQARAD, outperforming even the ensemble models of previous best solutions. Moreover, our solution provides attention maps which help in model interpretability. The code is available at https://github.com/VirajBagal/MMBERT
\end{abstract}

Index Terms - medical VQA, multimodal BERT, vision and language

\section{INTRODUCTION AND RELATED WORK}

Visual question answering (VQA) on medical images aspires to build models that can answer diagnostically relevant natural language questions asked on medical images. It can provide valuable additional insights to medical professionals and can help the patients in the interpretation of their medical images. However, supervised learning algorithms require large labeled datasets for effective performance and a major drawback of VQA in the medical domain is the small size of existing datasets [1, 2, 3]. Since the annotations on medical images require the help of an expert, it is difficult to crowdsource and annotation cost is high. This motivates the usage of self-supervised pretraining methods.

Self-supervised pretraining of BERT-like architectures has been proven quite effective in Natural Language Processing (NLP) [4], vision and Language [5, 6] space. The solution we propose - a Multimodal Medical BERT (MMBERT) is inspired

${ }^{\star}$ Equal Contribution. Order decided by coin toss. Work done during an internship at IIIT Hyderabad.
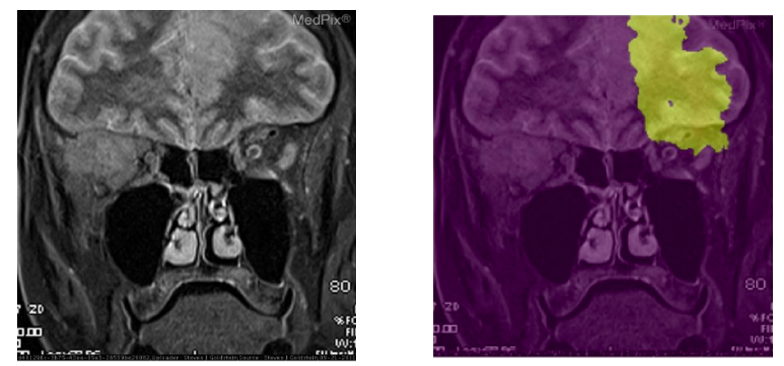

Question: What imaging modality was used? Answer: MR-T2 Weighted

Fig. 1: Example illustrating the attention map from our MMBERT model. For the given question, the model attends to grey matter, white matter and cerebrospinal fluid (CSF) and predicts the correct answer - 'MR-T2 Weighted'.

by these approaches. We first pretrain our MMBERT model on a set of medical images and their corresponding captions for the masked LM task. Later this model is finetuned for the VQA task.

Yan et al. [7] who are the winners of the VQA-Med 2019 challenge, use a Convolutional Neural Network (CNN) and BERT to extract image and question features respectively, followed by co-attention to fuse these features and a decoder to predict the answers. Ren et al. [8] propose a model called CGMVQA that uses a multimodal transformer architecture, similar to the proposed MMBERT. Zhan et al.[9] use a conditional reasoning framework for medical VQA on VQA-RAD dataset and they train a model separately for both the openended and closed-ended questions in the dataset.

Although the aforementioned methods obtain effective results, they do not use existing large multimodal medical datasets to learn better image and text representations. Our approach takes this into account and achieves new stateof-the-art performance on two medical VQA datasets. Our MMBERT, even with a single model for both the type of questions, yields better results than all the previous models on VQA-RAD. It also achieves a 5\% improvement in Accuracy over the previous state-of-the-art model on VQA-Med 2019 dataset. Moreover, our model provides attention maps and as shown in Fig 11, it focuses on correct region (grey and white 


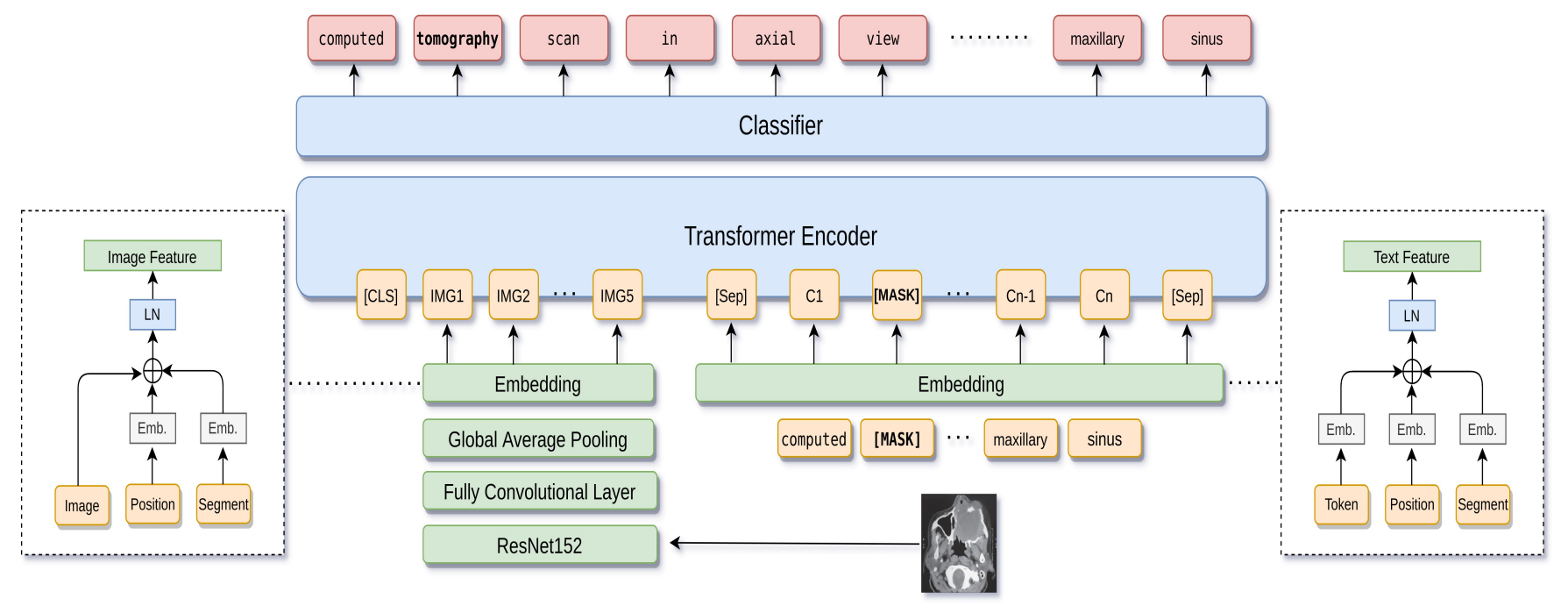

Fig. 2: Model architecture for MLM Pretraining task with image features on image caption data. To facilitate distinguishibility, we use embeddings of 0 and 1 as segment embeddings for image and text features respectively. We use embeddings of enumeration of image and text features as position embeddings for image and text respectively. The image features are extracted from ResNet152 and passed through an embedding layer. The caption is tokenized and the keywords are masked with [Mask] tokens. The text embeddings are obtained by combining input, position and segment embeddings. The final embedding is passed through a transformer encoder. The encoder outputs are then passed to a classifier which predicts the masked words.

matter difference) to predict the modality of the image.

\section{METHOD}

Transfer learning is quite popular in machine learning. However, a shift in image data distribution might result in sub-optimal performance when using pretrained weights from general domain. Moreover, there are changes in cooccurences of words in the medical text compared to the general domain text. These factors motivate the need for learning semantic representations of medical images and texts from scratch. Owing to the attention operation, we use Transformer encoder for learning effective representations.

\subsection{Self-Attention}

Self-Attention allows attention to intra-modality and intermodality features, thus enhancing the semantics of the intermediate representations. It involves mapping a query vector to the weighted addition of the value vectors where the weights are obtained by scaling the dot product of the query and the key vectors [10]. The query, key and value vectors are represented together in the matrices $Q, K$ and $V$ respectively. The dot product of $Q$ and $K$ is scaled inversely by $\sqrt{d_{k}}$, where $d_{k}$ is the dimension of query and key vectors.

$$
\operatorname{Attention}(Q, K, V)=\operatorname{softmax}\left(\frac{Q K^{T}}{\sqrt{d_{k}}}\right) V
$$

Instead of performing a single self-attention, the Transformer
Encoder performs multiple self-attentions (multi-head attention) in parallel and concatenates the output. Multi-head attention provides better representations by attending to different representation subspaces at different positions.

\subsection{Pretraining}

A schematic of the MMBERT pretraining is shown in Figure 2. For image features, similar to the CGMVQA [8] we use ResNet152 [12] and extract features from different convolution layers. This helps in retaining information from different resolutions. We use BERT Wordpiece tokenizer [4] for text tokenization. The sequence of 5 image features and the caption token embeddings together are provided as input to the BERTlike model. Unlike BERT $\mathrm{BASE}_{\mathrm{BSE}}$ [4] our model has only 4 BERT Layers and a total of 12 attention heads.

We use masked language modeling with image features as the pretraining task. In masked language modeling with image features, the task is to predict the original token in place of a [MASK] token with the usage of not only the accompanying text but also the image features. To ensure that the model learns to predict medical words from the context, we mask only medical keywords (provided with the dataset) from the captions and leave the common words untouched.

\subsection{Finetuning}

We load the model with weights from pretraining and finetune it further on the train split of the respective medical VQA dataset. Instead of using [CLS] (Classification) token repre- 


\begin{tabular}{|c|c|c|c|c|c|c|c|c|c|c|c|c|c|}
\hline \multirow[t]{2}{*}{ Method } & \multirow{2}{*}{$\begin{array}{c}\text { Dedicated } \\
\text { Models }\end{array}$} & \multicolumn{2}{|c|}{ Modality } & \multicolumn{2}{|c|}{ Plane } & \multicolumn{2}{|c|}{ Organ } & \multicolumn{2}{|c|}{ Abnormality } & \multicolumn{2}{|c|}{ Yes/No } & \multicolumn{2}{|c|}{ Overall } \\
\hline & & Acc. & BLEU & Acc. & BLEU & Acc. & BLEU & Acc. & BLEU & Acc. & BLEU & Acc. & BLEU \\
\hline VGG16+BERT [7] & - & - & - & - & - & - & - & - & - & - & - & 62.4 & 64.4 \\
\hline CGMVQA [8] & $\checkmark$ & 80.5 & 85.6 & 80.8 & 81.3 & 72.8 & 76.9 & 1.7 & 1.7 & 75.0 & 75.0 & 60.0 & 61.9 \\
\hline CGMVQA Ens. [8] & $\checkmark$ & 81.9 & 88.0 & 86.4 & 86.4 & 78.4 & 79.7 & 4.40 & 7.60 & 78.1 & 78.1 & 64.0 & 65.9 \\
\hline MMBERT General & $x$ & 77.7 & 81.8 & 82.4 & 82.9 & 73.6 & 76.6 & 5.20 & 6.70 & 85.9 & 85.9 & 62.4 & 64.2 \\
\hline MMBERT NP & $\checkmark$ & 80.6 & 85.6 & 81.6 & 82.1 & 71.2 & 74.4 & 4.30 & 5.70 & 78.1 & 78.1 & 60.2 & 62.7 \\
\hline MMBERT Exclusive & $\checkmark$ & 83.3 & 86.2 & 86.4 & 86.4 & 76.8 & 80.7 & 14.0 & 16.0 & 87.5 & 87.5 & 67.2 & 69.0 \\
\hline
\end{tabular}

Table 1: Results on VQA-Med 2019 dataset. Our method outperforms all previous methods that include methods with ensemble models in overall Accuracy and BLEU score. NP and Ens. refer to non-pretrained and ensemble models respectively.

\begin{tabular}{lcccc}
\hline \multicolumn{1}{c}{ Method } & Dedicated & \multicolumn{3}{c}{ Accuracy } \\
\cline { 3 - 5 } & Models & Open & Closed & Overall \\
\hline MEVF+SAN [11] & - & 40.7 & 74.1 & 60.8 \\
MEVF+BAN [11] & - & 43.9 & 75.1 & 62.7 \\
CR [9] & $\checkmark$ & 60.0 & $\mathbf{7 9 . 3}$ & 71.6 \\
\hline MMBERT General & $\boldsymbol{X}$ & $\mathbf{6 3 . 1}$ & 77.9 & $\mathbf{7 2 . 0}$ \\
\hline
\end{tabular}

Table 2: Results on VQA-RAD dataset. Our method with single model for both open-ended and closed-ended question types outperforms all previous methods including methods with dedicated models for each question type in overall Accuracy.

sentation from the last layer of the Transformer, we average the representation of each token obtained from the last layer and further pass it through dense layers for classification.

\section{EXPERIMENTS AND RESULTS}

\subsection{Data}

Radiology objects in Context (ROCO) [13] dataset contains over 81,000 radiology images with several medical imaging modalities. For pretraining, we use all the images, their corresponding captions and use the keywords for masking. VQAMed 2019 [2] is a challenge dataset introduced as part of the ImageCLEF-VQA Med 2019 challenge. It contains radiology images and has four main categories of questions: Modality, Plane, Organ system and Abnormality. All the samples having Yes/No as the ground truth are considered as Yes/No category. The dataset includes a training set of 3200 medical images with 12,792 Question-Answer (QA) pairs, a validation set of 500 medical images with 2000 QA pairs and a test set of 500 medical images with 500 QA pairs. VQA-RAD has 315 images and 3515 questions of 11 types. 58\% of questions are close-ended while the rest are open-ended.

\subsection{Experiments}

In our study, we primarily experiment with three different settings for the MMBERT: (i) MMBERT General: a model pretrained on ROCO and finetuned on all samples in the train split of the respective VQA dataset (ii) MMBERT Exclusive: an initial model pretrained on ROCO, which is further finetuned sep- arately for different question categories. For example in case of VQA-Med 2019, we learn 5 different models, one for each question category and (iii) MMBERT Non-Pretrained (NP): Dedicated models for each question category but without pretraining on the ROCO dataset. At the time of the inference, for settings where there are dedicated models for each question category we first predict the question category using a BERT $_{\text {BASE }}$ classifier.

We train the models on a single NVIDIA RTX 2080Ti GPU. For pretraining and finetuning, we resize all images to $224 \times 224$. We use image crop, rotation and color jitter for augmentation. For pretraining, we optimize the loss using Adam optimizer [14] with learning rate $2 e-5$ and reduce the learning rate by a factor of 0.1 if the validation loss does not improve for 5 consecutive epochs. For finetuning, we use the Adam optimizer, learning rate of $1 e-4$ and reduce the learning rate by a factor of 0.1 if validation loss does not improve for 10 consecutive epochs.

\subsection{Results and Analysis}

We use Accuracy and BiLingual Evaluation Understudy (BLEU) score to evaluate the VQA performance. BLEU score is the percentage of uniformly weighted 4-grams in the predicted answer that are shared with the ground truth. Table 1 reports results on the VQA-Med 2019 dataset. Our MMBERT Exclusive achieves state-of-the-art results on the overall Accuracy and BLEU score, even surpassing CGMVQA Ens. which is an ensemble of 3 dedicated models for each category. Even our MMBERT General performs better than the CGMVQA Ens. on the Abnormality and Yas/No categories. Additionally, our MMBERT General outperforms single dedicated CGMVQA models in all categories but Modality.

In the Organ category, MMBERT Exclusive outperforms CGMVQA Ens. in BLEU but not in Accuracy. BLEU score is calculated by counting matching 1-gram in the predicted answer to the 1-gram in the ground truth. The comparison is made regardless of the order. This suggests that even though our model couldn't predict perfectly right answers, it could predict more answers close to the ground truth than the CGMVQA Ens. We find the opposite behaviour in the Modality category. When compared to MMBERT NP, we find that the pretraining increases the Accuracy and BLEU score by 7.2 

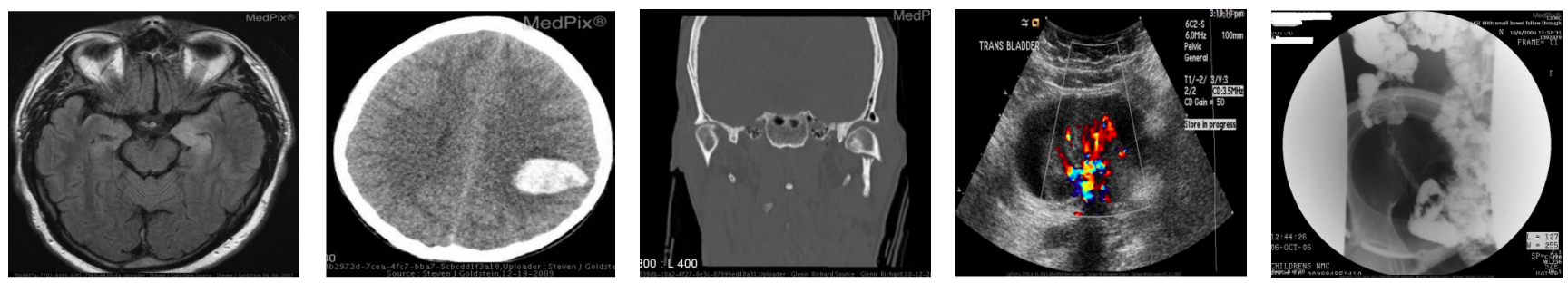

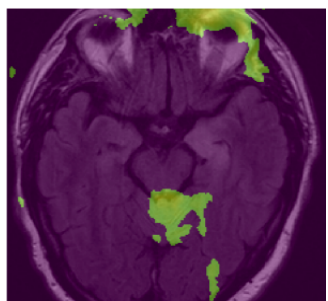

C: Organ

Q: What organ is the image of?

GT: skull and contents

ME: skull and contents

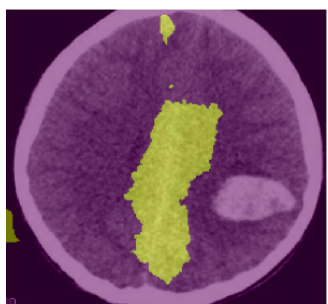

C: Plane

Q: What is the plane of the image?

GT: axial ME: axial

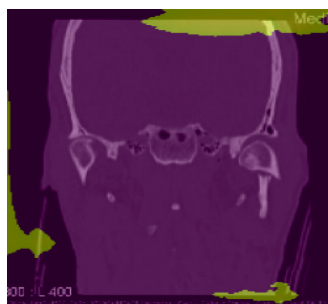

C: Yes/No

Q: Is this an MRI image? GT: no

ME: no

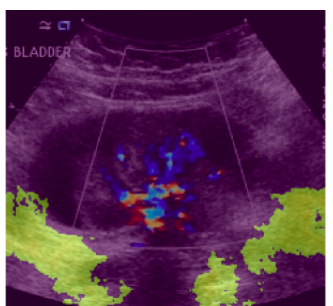

C: Modality

Q: What imaging method was used?

GT: us-d - doppler ultrasound

ME: us - ultrasound

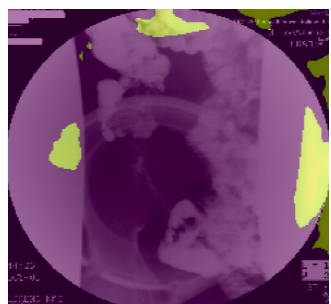

C: Abnormality

Q: What is abnormal in the image?

GT: crohn's disease

ME: fluoroscopic evaluation of small bowel in crohn's ileitis

Fig. 3: ME and GT refers to MmBERT Exclusive \& Ground Truth. The bottom row comprises attention maps for the corresponding top row images. In the Organ and Yes/No category, the model rightly attends to the bony part and soft tissue content to predict the right answer. In the Plane category, the model attends to the longitudinal fissure that is the key visual cue of the axial plane. The model fails to attend to the visual cue of doppler effect (colorful regions) in the Modality category. The Abnormality model surprisingly predicts a better answer than the ground truth by simultaneously predicting the modality, organ and abnormality.

and 9 points respectively.

Table 2 reports results on the VQA-RAD dataset. MMBERT General, which is a single model for both the question types in the dataset, outperforms the existing approaches including the ones which have a dedicated model for each question type.

\subsection{Qualitative Analysis}

Fig. 3 shows the category-wise qualitative results from MMBERT Exclusive. The top row comprises the original images while the bottom row comprises the attention maps obtained from our model. The attention maps highlight the regions in the image which contribute the most to the prediction. In the Organ and Yes/No category, the model rightly attends to the skull (the bony part) and its contents (brain tissues) to predict the right answer. In the Plane category, the model attends to the longitudinal fissure which is the key visual cue in identifying the axial plane as it separates the brain into Right and Left hemispheres. In the Modality category, the model attends to the soft tissue and fluid part of the image and is able to correctly predict that it is an ultrasound image. However, it fails to attend to the visual cue of the Doppler (the colour re- gion) and hence fails to correctly answer. Surprisingly, in the case of Abnormality category, for the attention map resulting for the "Fluoroscopic evaluation of small bowel in Crohn's ileitis" prediction, our model predicts a better answer than the ground truth. Here, it is simultaneously predicting the modality (fluoroscopy), organ (bowel) and the abnormality (Crohn's ileitis).

Medical experts find it difficult to make a correct diagnosis of abnormalities from a single image. They often resort to multiple sections (slices), planes, and other evidences. On closely analyzing our results we see that our model predicts abnormalities which could have also been a differential diagnosis for a human expert. However, our quantitative evaluation protocol does not take this into consideration.

\section{CONCLUSION}

In this work, we prospose to pretrain Multimodal Medical BERT (MMBERT) on ROCO dataset with masked language modeling using image features for medical VQA. We finetune it on VQA-RAD and VQA-Med 2019 datasets and achieve new state-of-the-art results on these datasets. Moreover, qualitative results show that our models can rightly attend to the 
image regions for prediction.

\section{COMPLIANCE WITH ETHICAL STANDARDS}

This research study was conducted retrospectively using human subject data made available in open access by O. Pelka et al.[13], Asma Ben Abacha et al.[2] and Lau et al.[1]. Ethical approval was not required as confirmed by the license attached with the open access data.

\section{ACKNOWLEDGMENTS}

No funding was received for conducting this study. The authors have no relevant financial or non-financial interests to disclose.

\section{REFERENCES}

[1] Lau et al., "A dataset of clinically generated visual questions and answers about radiology images," Scientific data, 2018.

[2] Abacha et al., "VQA-Med: Overview of the Medical Visual Question Answering Task at ImageCLEF 2019," in CLEF 2019 Working Notes, 2019, CEUR Workshop Proceedings.

[3] Xuehai He, Yichen Zhang, Luntian Mou, Eric Xing, and Pengtao Xie, "PathVQA: 30000+ questions for medical visual question answering," arXiv preprint arXiv:2003.10286, 2020.

[4] Devlin et al., "BERT: Pre-training of deep bidirectional transformers for language understanding," in NAACL, 2019.

[5] Jiasen Lu, Dhruv Batra, Devi Parikh, and Stefan Lee, "Vilbert: Pretraining task-agnostic visiolinguistic representations for vision-and-language tasks," in NeurIPS, 2019.

[6] Yen-Chun et al., "UNITER: Universal image-text representation learning," in $E C C V, 2020$.

[7] Yan et al., "Zhejiang university at ImageCLEF 2019 visual question answering in the medical domain.," in CLEF (Working Notes), 2019.

[8] Fuji Ren et al., "CGMVQA: A New Classification and Generative Model for Medical Visual Question Answering," in IEEE Access, 2020.

[9] Zhan et al., "Medical visual question answering via conditional reasoning," in ACM, 2020.

[10] Vaswani et al., "Attention is all you need," in Neural Information Processing Systems (NIPS), 2017.

[11] Nguyen et al., "Overcoming data limitation in medical visual question answering," in MICCAI, 2019.

[12] He et al., "Deep residual learning for image recognition," in CVPR, 2016.

[13] Pelka et al., "Radiology objects in context (ROCO): A multimodal image dataset," in Intravascular Imaging and Computer Assisted Stenting and Large-Scale Annotation of Biomedical Data and Expert Label Synthesis, 2018.
[14] Diederik P. Kingma and Jimmy Ba, "Adam: A method for stochastic optimization," in 3rd International Conference on Learning Representations, ICLR 2015, San Diego, CA, USA, May 7-9, 2015, Conference Track Proceedings, Yoshua Bengio and Yann LeCun, Eds., 2015. 